OPEN ACCESS

Edited by:

Ulrich Blank,

Institut National de la Santé et de la

Recherche Médicale

(INSERM), France

Reviewed by:

Mario Sanchez-Borges,

El Ávila Clinic, Venezuela

Andreas Kerstan,

University of Wuerzburg, Germany

*Correspondence:

Margitta Worm

margitta.worm@charite.de

tThese authors have contributed equally to this work

¥These authors share authorship

\$Participating centers can be found under www.anaphylaxie.net

Specialty section: This article was submitted to Molecular Innate Immunity, a section of the journal

Frontiers in Immunology

Received: 15 January 2019 Accepted: 20 March 2019 Published: 24 April 2019

Citation: Aurich S, Dölle-Bierke S, Francuzik W, Bilo MB, Christoff G, Fernandez-Rivas

M, Hawranek T, Pföhler C, Poziomkowska-Gessicka I, Renaudin J-M, Oppel E, Scherer K, Treudler R and Worm M (2019) Anaphylaxis in Elderly Patients-Data From the

European Anaphylaxis Registry. Front. Immunol. 10:750. doi: 10.3389/fimmu.2019.00750

\section{Anaphylaxis in Elderly Patients-Data From the European Anaphylaxis Registry}

\author{
Stefanie Aurich ${ }^{1 \dagger}$, Sabine Dölle-Bierke ${ }^{2 \dagger}$, Wojciech Francuzik ${ }^{2}$, Maria Beatrice Bilo ${ }^{3}$, \\ George Christoff ${ }^{4,5}$, Montserrat Fernandez-Rivas ${ }^{6}$, Thomas Hawranek ${ }^{7}$, Claudia Pföhler ${ }^{8}$, \\ Iwona Poziomkowska-Gessicka ${ }^{9}$, Jean-Marie Renaudin ${ }^{10}$, Eva Oppel ${ }^{11}$, Kathrin Scherer ${ }^{12}$, \\ Regina Treudler ${ }^{1 \ddagger}$ and Margitta Worm ${ }^{2 * \neq}$ \\ on behalf of The Network for Online Registration of Anaphylaxis(NORA) ${ }^{\S}$
}

${ }^{1}$ Department of Dermatology, Venereology and Allergology, LICA-Comprehensive Allergy Center, University Hospital, Leipzig, Germany, ${ }^{2}$ Division of Allergology and Immunology, Department of Dermatology, Venereology and Allergology, Charité-Universitätsmedizin Berlin, Berlin Institute of Health, Corporate Member of Freie Universität Berlin,

Humboldt-Universität zu Berlin, Berlin, Germany, ${ }^{3}$ Allergy Unit, Department of Internal Medicine, University Hospital Ospedall Riuniti, Ancona, Italy, ${ }^{4}$ Faculty of Public Health, Medical University-Sofia, Sofia, Bulgaria, ${ }^{5}$ Allergy Outpatient Unit, Acibadem CityClinic, Medical Centre Tokuda, Sofia, Bulgaria, ${ }^{6}$ Allergy Department, Hospital Clinico San Carlos, Madrid, Spain,

${ }^{7}$ Department of Dermatology, University Hospital of the Paracelsus Private Medical University, Salzburg, Austria, ${ }^{8}$ Department of Dermatology, Venereology and Allergology, Saarland University Medical Center, Homburg, Germany, ${ }^{9}$ Clinical Allergology Department, Pomeranian Medical University in Szczecin, Szczecin, Poland, ${ }^{10}$ Presidency, Allergy Vigilance Network, Vandoeuvre les Nancy, France, ${ }^{11}$ Department of Dermatology and Allergy, University Hospital (LMU), Munich, Germany, ${ }^{12}$ Division of Allergy, Department of Dermatology, University of Basel, Basel, Switzerland

Background: Elicitors and symptoms of anaphylaxis are age dependent. However, little is known about typical features of anaphylaxis in patients aged 65 years or more.

Methods: The data from the Network for Online Registration of Anaphylaxis (NORA) considering patients aged $\geq 65$ (elderly) in comparison to data from adults (18-64 years) regarding elicitors, symptoms, comorbidities, and treatment measures were analyzed.

Results: We identified 1,123 elderly anaphylactic patients. Insect venoms were the most frequent elicitor in this group $(0<0.001)$, followed by drugs like analgesics and antibiotics. Food allergens elicited less frequently anaphylaxis $(p<0.001)$. Skin symptoms occurred less frequently in elderly patients $(77 \%, p<0.001)$. The clinical symptoms were more severe in the elderly (51\% experiencing grade III/IV reactions), in particular when skin symptoms $(p<0.001)$ were absent. Most strikingly, a loss of consciousness (33\%, $p<0.001$ ) and preexisting cardiovascular comorbidity (59\%, $p<0.001$ ) were more prevalent in the elderly. Finally, adrenaline was used in $30 \%$ of the elderly (vs. 26\% in the comparator group, $p<0.001$ ) and hospitalization was more often required (60 vs. $50 \%, p<0.001$ ).

Discussion and Conclusion: Anaphylaxis in the elderly is often caused by insect venoms and drugs. These patients suffer more often from cardiovascular symptoms, receive more frequently adrenaline and require more often hospitalization. 
The data indicate that anaphylaxis in the elderly tends to be more frequently life threatening and patients require intensified medical intervention. The data support the need to recognize anaphylaxis in this patient group, which is prone to be at a higher risk for a fatal outcome.

Keywords: anaphylaxis, elderly, insect venom, drug hypersensitivity, adrenaline, hospitalization, loss of consciousness

\section{INTRODUCTION}

The incidence of anaphylaxis, which may occur at any age, is rising in Western countries $(1,2)$. While life expectancy is increasing, little is known about the characteristics of anaphylaxis in elderly patients (3). Existing data indicate age dependent differences with regard to elicitors, cofactors, and symptoms of anaphylaxis (4).

Overall, elderly patients have a higher risk for severe and fatal reactions (5).

The main elicitors of anaphylaxis are insect venoms, drugs and food items (6). While food items are the most frequent elicitors in children and young adults (7), drug anaphylaxis is more common in elderly patients (8). Patients above 65 were reported to be prone to develop cardiovascular symptoms more frequently (9). It is unknown whether this is due to the increased prevalence of cardiovascular diseases in this age group leading to limited cardiovascular compensation mechanisms (10), use of cardiovascular drugs (5), or both. Cofactors like exercise, drugs, alcohol, and stress are supposed to reduce the threshold of allergic reactions (11).

Recommendations for the emergency treatment of anaphylaxis are similar for all age groups and are supported by current guidelines (12). Some considerations and adaptions should be made in elderly patients. Although, the administration of adrenaline in anaphylactic patients with known or suspected cardiovascular diseases is not contraindicated, it might sometimes cause difficulties due to an increased coronary blood flow e.g., in patients with acute coronary syndrome (10). However, current guidelines clearly state that the benefit of adrenaline usage outweighs its risks through beneficial effects even in suspected anaphylaxis (12).

In this study, we analyzed data from adult patients differentiated by age from the European Anaphylaxis Registry registered between 2007 and 2017. The aim was to better understand anaphylaxis in patients $\geq 65$ years. Data regarding elicitors, symptoms, comorbidities, and emergency treatment were considered.

\section{METHODS}

\section{Setting and Design}

The European Anaphylaxis Registry collects information on anaphylactic reactions through a web based electronic data capture system as described previously (6). Participation of study centers is voluntary. Data for the current analysis were

Abbreviations: ICU, Intensive Care Unit. provided by tertiary referral centers specialized in allergology and/or dermatology in Germany, Switzerland, France, Austria, Spain, Italy, Bulgaria, and Poland. The study was approved by the Ethics Committee at Charité-Universitätsmedizin Berlin (the coordinating center) and by the local Ethics Committees.

\section{Data Source and Handling}

After completion of diagnostics, patients' data were retrieved from medical treatment, laboratory measurements, and emergency protocols as available. Using a pseudonym, the data were entered by trained study personnel into an online questionnaire in each study center. The questionnaire and data entry is described elsewhere (7). Data collected from July 2007 to March 2017 were included.

\section{Variables}

Age at reaction was categorized in two groups: patients aged 1864 years (adults), and patients 65 years and older (elderly). The gathered variables are described elsewhere (7).

\section{Statistical Analysis}

The analysis of the data was carried out using R. The ShapiroWilk's test was used to assess the normality of distribution of interval variables. Interval variables were expressed as mean \pm standard deviation. Distribution of categorical variables were expressed as percentages. Differences in categorical variables were tested using pairwise $\mathrm{Chi}^{2}$ tests with Holm's correction for multiple comparisons. The values $P<0.05$ were considered statistically significant.

\section{RESULTS}

The European Anaphylaxis Registry collected data of 10,203 cases between July 2007 and March 2017. Six thousand eight hundred ninety-one patients met the criteria of Ring and Messmer $\geq$ grade II and were aged $\geq 18$ years. The cases were registered from 78 study centers in seven countries: Germany $(n=4,474)$, Switzerland $(n=777)$, France $(n=529)$, Austria $(n=461)$, Spain $(n=267)$, Italy $(n=188)$, Bulgaria $(n=100)$, and Poland $(n=95)$.

Of the 6,891 cases, 5,768 were adults younger than 65 years and 1,123 were $\geq 65$ years old (Figures 1,2 ). The median age in elderly patients was 70 (65-93) years and 95\% of the cases are between 65 and 80 years (Figure 2B). In general, both adult groups comprised more females than males, but the elderly included a higher percentage of male patients in comparison to the comparator adult group $(p=0.005$, Table $\mathbf{1})$. 


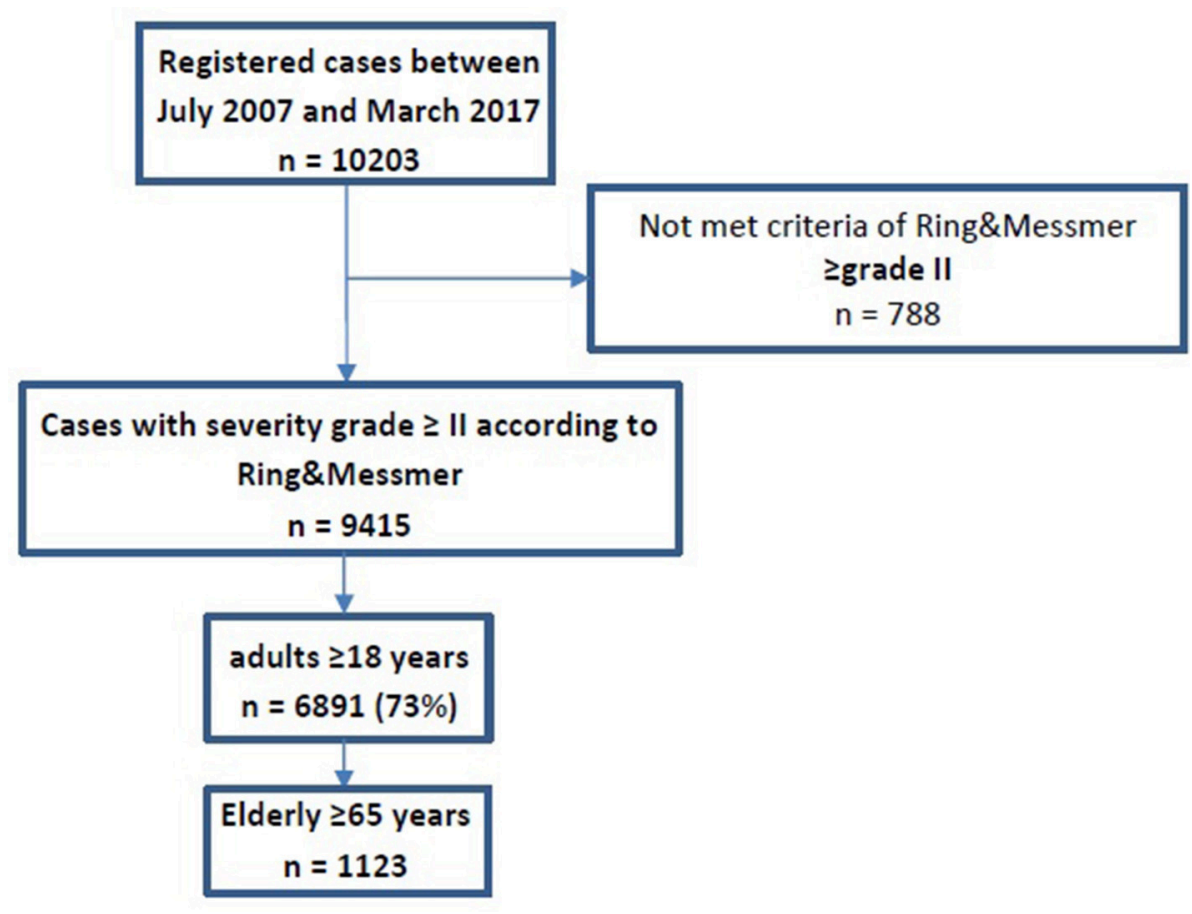

FIGURE 1 | Flow chart of study cohort.
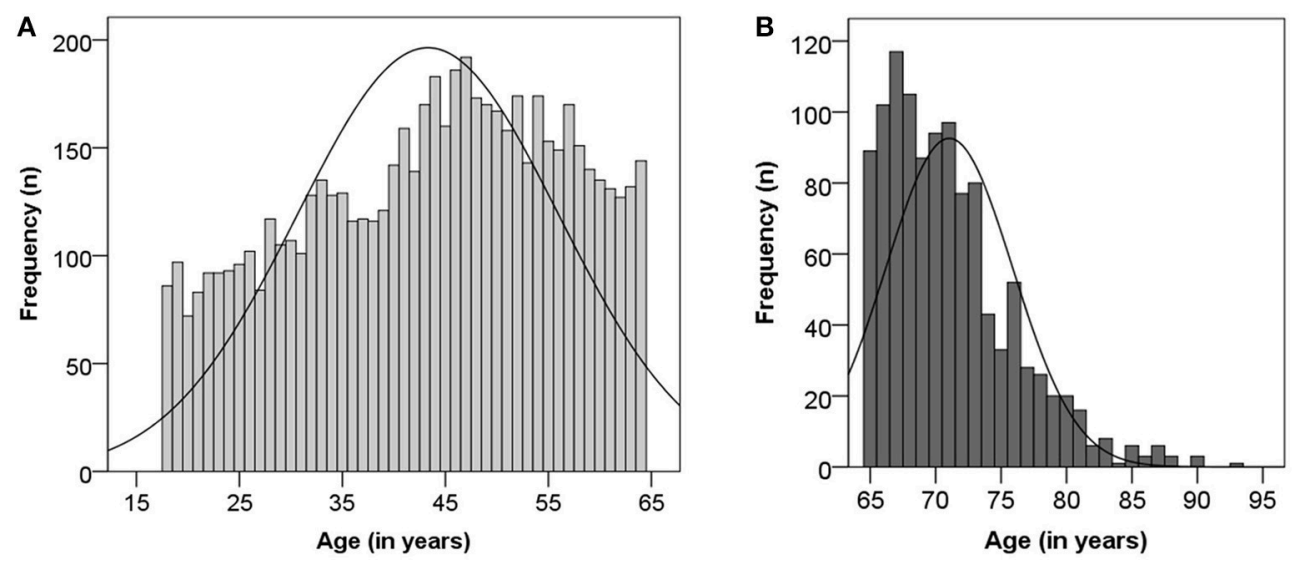

FIGURE 2 | Age distribution among adults (A) and the elderly (B).

The recent medical history in elderly patients is characterized by significantly more frequent cardiovascular diseases, thyroid and malignant diseases than in younger adults. In contrast, in the younger adult group, atopic diseases were significantly more common than in the elderly $(p<0.001)$.

Potential cofactors of any type (Table 1) were significantly more prevalent in elderly patients $(p<0.001)$ and there was a considerable association between the age and the concomitant drug intake, under which ace inhibitor, AT-2 antagonist, betablocker, acetylcholine, and proton pump inhibitor were taken into account (Table 1; $p<0.001$ ). Twenty-eight percentage of elderly patients reported a previous allergic reaction to the same elicitor. Of these 75\% reported a previous milder reaction, 19\% had a similar or even more severe reaction (data not shown).

\section{Insect Venom and Drugs Are Main Causes of Anaphylaxis in the Elderly}

Elicitors were more frequently identified in the elderly (79\%) than in the younger adult group (74\%; $p<0.001$; Table 2). Insect venom anaphylaxis was most prevalent in elderly patients $(n=$ 633 ; 56\%; $p<0.001$ ), with yellow jacket $(n=451,71 \%)$ being the cause in the majority of cases. Drugs were the second main causative agent of anaphylaxis across adults again being more prevalent in the elderly $(25 \% ; p=0.009)$. Analgesics [metamizole 
TABLE 1 | Characteristics of the elderly in comparison to the adult group.

\begin{tabular}{|c|c|c|c|}
\hline & $\begin{array}{c}\begin{array}{c}\text { Elderly } \\
(\geq 65 \text { years) }\end{array} \\
n(\%)\end{array}$ & $\begin{array}{c}\text { Adults } \\
\text { (18-64 } \\
\text { years) } \\
n(\%)\end{array}$ & Chi ${ }^{2}$ test \\
\hline TOTAL & $1,123(16)$ & $5,768(84)$ & \\
\hline Median age in years (min-max) & $70(65-93)$ & $45(18-64)$ & \\
\hline Male & $517(46)$ & 2,394 (42) & $p=0.005$ \\
\hline \multicolumn{4}{|l|}{ COMORBIDITIES } \\
\hline Atopic & $190(18)$ & 1,628 (30) & $p<0.001$ \\
\hline Cardiovascular & $641(60)$ & $960(18)$ & $p<0.001$ \\
\hline Mastocytosis & $39(4)$ & $136(3)$ & $\begin{array}{l}N S(p= \\
0.051)\end{array}$ \\
\hline Thyroid & $162(15)$ & $476(9)$ & $p<0.001$ \\
\hline Urticaria & $14(1)$ & $104(2)$ & NS \\
\hline Malignant disease & $63(6)$ & $97(2)$ & $p<0.001$ \\
\hline \multicolumn{4}{|l|}{ COFACTORS } \\
\hline Overall & $802(71)$ & 2,897 (54) & $p<0.001$ \\
\hline Drugs $\#$ & $614(57)$ & $991(18)$ & $p<0.001$ \\
\hline Physical exercise & $267(27)$ & $1,609(31)$ & $p=0.02$ \\
\hline Psychological stress & $84(8)$ & $418(7)$ & NS \\
\hline Alcohol & $48(5)$ & $326(7)$ & $\begin{array}{l}N S(p= \\
0.051)\end{array}$ \\
\hline Acute Infection & $33(3)$ & $177(3)$ & NS \\
\hline \multicolumn{4}{|c|}{ SEVERITY ACCORDING TO RING\&MESSMER } \\
\hline Grade II & $550(49)$ & $3,505(61)$ & $p<0.001$ \\
\hline Grade III & $525(47)$ & $2,105(37)$ & $p<0.001$ \\
\hline Grade IV & $48(4)$ & $158(3)$ & $p=0.023$ \\
\hline
\end{tabular}

In total 6,891 cases $\geq 18$ years old were analyzable until March 2017. Statistical calculations were performed with chi ${ }^{2}$ test including post-hoc corrections. Significant differences were given in comparison to adults between 18 and 64 years of age.

\#Drugs counted as cofactors were ace-inhibitor, AT-2-antagonist, beta-blocker, acetylcholine, and proton pump inhibitor.

( $n=19 ; 7 \%)$, diclofenac $(n=45 ; 16 \%)$, ibuprofen $(n=17 ; 6 \%)]$ and antibiotics [penicillin $(n=23 ; 8 \%)$, cephalosporins $(n=30$; $11 \%)$, gyrase inhibitors/ quinolones $(n=14 ; 5 \%)]$ were the most frequent single elicitors of drug anaphylaxis in the elderly. Food was a predominant elicitor in adults $(22 \%, p<0.001$; Table 2$)$. In the elderly only $11 \%$ of reactions were caused by food, with wheat (14\%) and hazelnut (13\%) as leading food allergens.

\section{Cardiovascular Symptoms Predominate in the Elderly}

Cardiovascular symptoms occurred frequently in elderly patients ( $80 \%$ compared to adults $75 \% ; p<0.001$ ). A major cardiovascular symptom was loss of consciousness (adults: $20 \%$; elderly $33 \%, p$ $<0.001$ ) while dizziness and tachycardia were more prevalent in adults (Figure 3D). Cardiac arrest occurred in 153 cases (3\% in elderly vs. $2 \%$ in adults). The skin was the most frequently involved organ system of all affected patients. However, there was a shift showing that elderly patients are less frequently affected (77\%) in comparison to younger adults $(83 \%$; $p<0.001$; Figure $3 \mathrm{~A}$ ). The severity of the reactions in elderly patients without skin symptoms was increased in comparison to the
TABLE 2 | Elicitor profile in the elderly in comparison to the younger adult group.

\begin{tabular}{|c|c|c|c|}
\hline & $\begin{array}{c}\begin{array}{c}\text { Elderly } \\
(\geq 65 \text { years) }\end{array} \\
n(\%)\end{array}$ & $\begin{array}{c}\text { Adults } \\
(18-64 \\
\text { years) } \\
n(\%)\end{array}$ & $C h i^{2}$ test \\
\hline TOTAL & $1,123(16 \%)$ & $5,768(84 \%)$ & \\
\hline Elicitor known & $883(79)$ & 4,242 (73) & $p<0.001$ \\
\hline INSECTS & $633(56)$ & $2,708(47)$ & $p<0.001$ \\
\hline Yellow jacket & $451(71)$ & 1,936 (72) & NS \\
\hline Bee & $97(15)$ & $512(19)$ & NS \\
\hline Hornet & $56(9)$ & $153(6)$ & $p<0.001$ \\
\hline DRUGS & $285(25)$ & 1,257 (22) & $p=0.009$ \\
\hline Analgesics & $103(36)$ & $354(36)$ & NS \\
\hline Antibiotics & $74(26)$ & $354(28)$ & NS \\
\hline Local anesthetics & $17(6)$ & $106(8)$ & NS \\
\hline x-ray (contrast agent) & $22(8)$ & $56(4)$ & $P=0.010$ \\
\hline PPI & $6(2)$ & $30(2)$ & NS \\
\hline Cardiovascular drugs & $5(1.8)$ & $8(0.6)$ & NS \\
\hline FOOD & $122(11)$ & 1,254 (22) & $p<0.001$ \\
\hline Wheat & $17(14)$ & $177(14)$ & NS \\
\hline Hazelnut & $16(13)$ & $84(7)$ & $p=0.015$ \\
\hline Shellfish & $15(12)$ & $123(10)$ & NS \\
\hline Celery & $7(6)$ & $74(6)$ & NS \\
\hline Soy & $6(5)$ & $78(6)$ & NS \\
\hline Peanut & $1(0.8)$ & $68(5)$ & $p=0.045$ \\
\hline IMMUNOTHERAPY (SIT) & $5(0.4)$ & $63(1.1)$ & $p=0.006$ \\
\hline
\end{tabular}

The elicitor was not specified in $423(6 \%)$ patients. Rare elicitors are not reported in detail. Statistical analysis was performed with Chi ${ }^{2}$ test including post-hoc corrections. Significant differences were given in comparison to adults between 18 and 64 years of age. Food items reported $<10$ times in the whole study are not listed in detail. PPI, proton pump inhibitor.

corresponding group of adults not suffering from skin symptoms $(p<0.001$, data not shown). Gastrointestinal symptoms occurred in a similar proportion in both adult groups (Figure 3B). The respiratory system was less frequently affected in the elderly (63\% compared to adults $70 \% ; p<0.001$ ), especially dyspnea (adults: 55\%; elderly 51\%; $p<0.001$; Figure 3C). Severe anaphylactic reactions including grade III $(47 \%)$ and grade IV (4\%) anaphylaxis were more prevalent in the elderly (Table 1).

\section{Increased Administration of Adrenaline in the Elderly}

First-line treatment was carried out by emergency physicians in $5,481(92 \%)$ of 5,971 patients who received first-line treatment. The administration rate of adrenaline by professionals in elderly patients was 30 and $26 \%$ in younger adults (Figure S1, across all severity grades; $p=0.043$ ). Hospitalization was required in 60 and $19 \%$ of elderly patients were treated in an intensive care unit (ICU) (Figure 4). Regardless the severity grade, the hospitalization and the ICU admission rates were higher in the elderly $(p<0.01)$. 

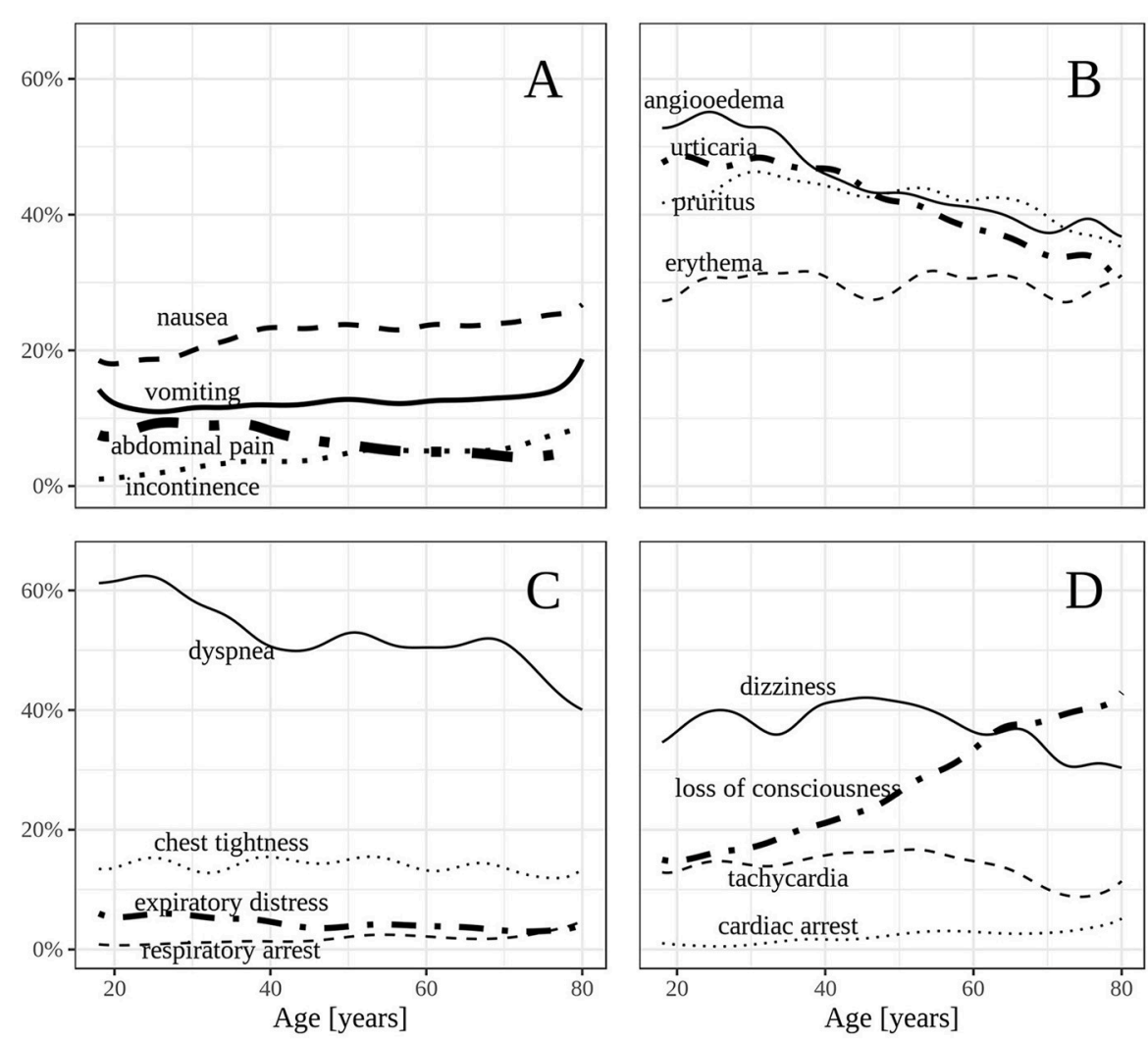

FIGURE 3 | (A-D) Frequency of selected symptoms according to age in adults.

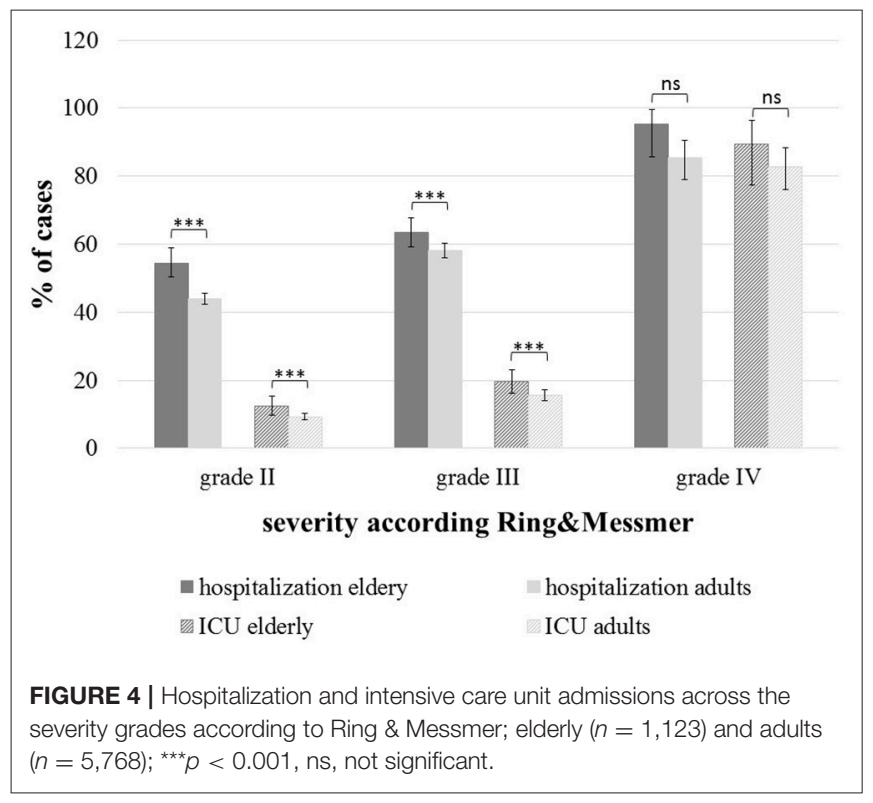

\section{DISCUSSION}

\section{Key Results}

This is the first large-scale analysis of anaphylactic patients including 1,123 cases aged 65 years or more. The European
Anaphylaxis Registry covers a heterogenic sample of patients across all age groups and a wide range of elicitors, from different European countries $(6,13)$.

The most frequent elicitor in both adult groups was insect venom, but elderly patients had significantly more insect elicited cases than younger adults (56 vs. $47 \%$ ). The data indicate that the clinical reactivity to insect venoms increases with age, although the individual exposure to insect stings might be comparable among adults, whereas one could speculate that retired elderly spend more time outdoor, e.g., in the garden. However, supposing comparable exposure, one reason for this observation might be the higher frequency of comorbidities, which may enhance the clinical response pattern particularly in patients with preexisting cardiovascular diseases increasing the risks for severe cardiovascular symptoms like tachyarrhythmia or syncope.

Moreover, we identified drugs as frequent elicitors in the elderly. This finding is in line with previous data from the registry and most likely associated with a higher drug intake in this patient group $(6,14)$. Also, drug interaction maybe relevant in this context. Another previous study analyzing patients $\geq 71$ years identified males to be predisposed to develop insect sting anaphylaxis whereas females suffered more often from drug anaphylaxis (15).

Furthermore, the distribution of elicitors differ by country (6). This observation is also reflected in the elderly patient group from 
different countries, e.g., drugs were the most frequent elicitors in Bulgaria and Spain and not insect venoms (data not shown).

The skin is the most frequently involved organ system in anaphylaxis as shown by many different studies including our own data (6). Most interestingly, the occurrence of skin symptoms in the elderly was significantly reduced compared to the younger adults. Moreover, this lack of skin symptoms was associated with more severe anaphylaxis in the group of the elderly patients. This phenomenon has been described previously and been attributed to the fast development of a severe circulatory impairment (16). Another assumption regarding the lack of skin symptoms and the onset of a severe reaction might be a delay of the diagnosis. A lack of skin symptoms leads to a significant reduced identification rate of anaphylaxis and consequently to delayed therapy (17). However, this would still not explain why skin symptoms are less frequent in this patient group, but may also be attributed to a decreased vascularization of the skin in higher age groups. A final hypothesis might be the intake of drugs affecting skin reactivity like betablockers or neuroleptics.

Cardiovascular manifestations of anaphylaxis occurred more frequently in our elderly patient cohort. Most interestingly, loss of consciousness was the predominant symptom in this patient group and has been observed by us in a previous analysis (18). Considering the overall adult cohort, most patients suffering from cardiovascular symptoms presented with dizziness and hypotension.

Adrenaline is the first treatment of choice in anaphylaxis and recommended by several international guidelines (12). However, data from others as well as our group indicate that most patients are not treated according to guidelines. By contrast, professional first-line treatment frequently includes the use of corticosteroids and antihistamines but less frequently adrenaline, especially in Germany (19). The data from this study show a significant impact of an increased age and the higher usage rate of adrenaline among all severity grades of the reaction. This finding is striking considering the fact that the group of elderly patients suffered more frequently from cardiovascular diseases and took more concomitant medication (10). The higher usage of adrenaline in the elderly might also be due to the higher percentage of patients experiencing grade IV reactions, since adrenaline is often used in cardiopulmonary resuscitation. Even though adrenaline was applied more often in the elderly than in younger adults investigated here, still $<1$ out of three patients received adrenaline as first line treatment. Adrenaline was mostly applied intravenously by professionals in the elderly (data not shown) not fulfilling the recommendations of the current guidelines for the management of anaphylaxis (12). The administration of intramuscular adrenaline is the route of first choice, because the risk of cardiovascular side effects is considerably lower (20).

This data indicate the need of educational measures regarding the acute treatment of anaphylaxis not only among patients but also among professionals. In particular, in emergency medical professionals the recognition of barriers against the use of adrenaline by an intramuscular route requires more attention. Recently, also guidelines from the resuscitation council are in favor of the first line intramuscular route and are an important step forward to change current clinical practice (21).

Finally, we observed increased hospital admission rates and treatment in ICU in elderly patients. Such an observation has been reported in previous studies from the US $(9,22)$. Most studies explain this phenomenon attributed to more severe reactions in elderly patients (8). However, our data suggest, that the hospital and the ICU admission rate was significantly higher in the elderly independent from the severity grade of anaphylaxis. Therefore, we propose that mostly due to comorbidities the patients are more often hospitalized, whether biphasic anaphylaxis occurs more frequently in the elderly patients is not known, but should be analyzed in more detail in future studies.

\section{Strength and Limitations}

We report on a large group of elderly European anaphylactic patients covering a 10-year observational period. The webbased assessment tool for registration covers several aspects of an anaphylactic reaction in a standardized manner, including elicitors, symptoms, co-factors, and emergency treatment. The online questionnaire was consistently revised and piloted (6).

The data are limited to patient records and not representative for a population as only patients are registered when seen for an allergy assessment after a given reaction.

\section{CONCLUSION}

In this large-scale description of anaphylaxis in patients aged 65 years and older, we report on characteristic age-dependent elicitors, comorbidities, symptoms, and emergency treatment. Features of anaphylaxis in patients aged 65 or more years differ from those of the younger adult group. The elderly are more likely to have anaphylaxis without skin symptoms which is associated with more difficulties to make diagnosis of anaphylaxis and more severe reactions. Moreover, the risk for insect venom anaphylaxis raises with age. The hospitalization and ICU admission rate were higher in elderly patients independent from the severity grade indicating that such patients require intensified medical intervention.

This study is one of the first comprehensive studies on patients $\geq 65$ years who experienced anaphylaxis. It contributes to a better management and identification of specific medical needs in an aging population.

\section{ETHICS STATEMENT}

The study was approved by the Ethics Committee at CharitéUniversitätsmedizin Berlin (the coordinating center) and by the local Ethics Committees.

\section{AUTHOR CONTRIBUTIONS}

SA and SD-B acquired data and were responsible for the concept and design of the manuscript, for interpretation of the 
data, writing and final approval of the manuscript. SD-B and WF were responsible for the data analysis and interpretation. MB, GC, MF-R, TH, CP, IP-G, J-MR, EO, and KS acquired data, revised the manuscript critically for important intellectual content, and approved the final manuscript. MW and RT performed conceptualization of the study and were responsible for supervised interpretation of the data, writing, and final approval of the manuscript.

\section{FUNDING}

The European Anaphylaxis Registry was supported by the Network for Online-Registration of Anaphylaxis NORA e. V.

\section{ACKNOWLEDGMENTS}

We thank all patients for their support of providing data on the occurrence of anaphylaxis to the Anaphylaxis Registry. We would like to thank the study personnel of every single center for data entry: J. Grünhagen (Berlin, Germany), K. Beyer (Berlin, Germany), A. Möser (Jena, Germany), T. Fuchs (Göttingen, Germany), F. Ruëff, M. Knop (Munich, Germany), B. Wedi (Hanover, Germany), H. Dickel (Bochum, Germany), H. Merk (Aachen, Germany), U. Hillen (Essen, Germany), A. Bauer (Dresden, Germany), N. Wagner (Erlangen, Germany), E. Rietschel (Cologne, Germany), L. Klimek, O. Pfaar (Wiesbaden, Germany), N. Reider (Innsbruck, Germany), W. Aberer (Graz, Austria), A. Köhli (Zurich, Switzerland), F. Riffelmann (Schmallenberg, Germany), B. Kreft (Halle, Germany), K. Nemat (Dresden, Germany), T. Kinaciyan (Vienna, Austria), R. Brehler (Münster, Germany), J. Witte (Hamburg, Germany), N. Hunzelmann und I. Huseynow (Cologne, Germany), T. Bieber (Bonn, Germany), U. Rabe (Treuenbritzen, Germany), P. Schmid-Grendelmeier (Zurich, Switzerland), W. Brosi (Würzburg, Germany), S. Nestoris (Lippe-Lemgo, Germany), R. Lang (Salzburg, Austria), R. Bruns (Greifswald, Germany), S. Lehmann (Aachen, Germany), L. Lange (Bonn, Germany), G. Hansen (Hanover, Germany), S. Becker, N. Krecké (Homburg, Germany), E. Varga (Graz, Austria), Z. Szepfalusi (Vienna, Austria), P. Eng (Aarau, Switzerland), P. Eng (Lucerne, Switzerland), T. Reese (Rheine, Germany), M. Polz (Rüsselsheim, Germany), S. SchweitzerKrantz (Düsseldorf, Germany), H. Rebmann (Tübingen, Germany), G. Stichtenoth (Lübeck, Germany), S. Theis (Schwedt, Germany), I. Yildiz (Neumünster, Germany), M. Gerstlauer (Augsburg, Germany), A. Nordwig (Dresden,

\section{REFERENCES}

1. Decker WW, Campbell RL, Manivannan V, Luke A, St Sauver JL, Weaver A, et al. The etiology and incidence of anaphylaxis in Rochester, Minnesota: a report from the Rochester Epidemiology Project. J Allergy Clin Immunol. (2008) 122:1161-5. doi: 10.1016/j.jaci.2008.09.043

2. Tejedor Alonso MA, Moro Moro M, Múgica García MV, Esteban Hernández J, Rosado Ingelmo A, Vila Albelda $\mathrm{C}$, et al. Incidence of anaphylaxis in the
Germany), T. Spindler (Wangen im Allgäu, Germany now Davos, Switzerland), I. Neustädter (Fürth, Germany), C. Stadlin (Zurich, Switzerland), M. Bücheler (Bonn, Germany), S. Volkmuth (Velbert, Germany), J. Fischer (Tübingen, Germany), A. Henschel (Berlin, Germany), S. Plank-Habibi (Alzenau, Germany), B. Schilling (Passau, Germany), A. Kleinheinz (Buxtehude, Germany), K. Schäkel (Heidelberg, Germany), N. Papadopoulus (Athen, Greece), M. Kowalski (Lodz, Poland), K. Solarewicz-Madajek (Wroclaw, Poland), C. Körner-Rettberg (Bochum, Germany), S. Tsheiller (Allergy vigilance network, France), T. Mustakov (Sofia, Bulgaria), K. Hartmann (Lübeck, Germany), C. Kemen (Hamburg, Germany), F. Prenzel (Leipzig, Germany), C. Ebner (Vienna, Austria), S. Haak (Oldenburg, Deutschland), V. Cardona (Barcelona, Spain), S. Hämmerling (Heidelberg, Germany), B. Garcia (Pamplona, Spain), N. Cabañes Higuero (Toledo, Spain), A. Vega Castro (Guadalajara, Spain), S. Büsing (Osnabrück, Germany), U. Klettke (Berlin, Germany), C. Virchow (Rostock, Germany), U. Müller (Borstel, Germany), S. Müller und T. Jakob (Freiburg, Germany), H. Straube (Darmstadt, Germany), C. Vogelberg (Dresden, Germany), F. Knöpfel (Norderney, Germany), J. Hourihane, I. Maris (Cork, Ireland), B. Rogala (Silesia, Poland), A. Montoro (Madrid, Spain), A. Brandes (Frankfurt/Oder, Germany), A. Muraro (Padua, Italy), T. Buck, J. Büsselberg (Hanover-Misburg, Germany), N. Zimmermann (Potsdam, Germany), D. Hernandez (Valencia, Spain), P. Minale (Genua, Italy), J. Niederwimmer und B. Zahel (Linz, Austria), A. Fiocchi (Rome, Italy), A. Reissig (Gera, Germany), F. Horak (Vienna, Austria), S. Meller (Düsseldorf, Germany), F. Eitelberger (Wels, Austria), H. Ott (Hanover, Germany), R. Asero (Milan, Italy), F. Hermann, S. Zeidler (St. Augustin, Deutschland), S. Pistauer (Sylt/Westerland, Germany), M. Geißler (Ribnitz-Damgarten, Germany), E. Cichoka-Jarosz (Krakow, Poland), L. Ensina (São Paulo, Brazil), A. Plaza Martin (Barcelona, Spain), J. Meister (Aue, Germany), P. Stock, S. Hompes (Hamburg, Germany), S. Stieglitz (Wuppertal, Germany).

We acknowledge support from the German Research Foundation (DFG) and the Open Access Publication Fund of Charité - Universitätsmedizin Berlin.

\section{SUPPLEMENTARY MATERIAL}

The Supplementary Material for this article can be found online at: https://www.frontiersin.org/articles/10.3389/fimmu. 2019.00750/full\#supplementary-material 
5. Francuzik W, Dölle S, Worm M. Risk factors and treatment of refractory anaphylaxis - a review of case reports. Expert Rev Clin Immunol. (2018) 14:307-14. doi: 10.1080/1744666X.2018.1450140

6. Worm M, Moneret-Vautrin A, Scherer K, Lang R, Fernandez-Rivas M, Cardona V, et al. First European data from the network of severe allergic reactions (NORA). Allergy. (2014) 69:1397-404. doi: 10.1111/all.12475

7. Grabenhenrich LB, Dölle S, Moneret-Vautrin A, Köhli A, Lange L, Spindler T, et al. Anaphylaxis in children and adolescents: the European Anaphylaxis Registry. J Allergy Clin Immunol. (2016) 137:1128-37.e1. doi: 10.1016/j.jaci.2015.11.015

8. Turner PJ, Gowland MH, Sharma V, Ierodiakonou D, Harper N, Garcez $\mathrm{T}$, et al. Increase in anaphylaxis-related hospitalizations but no increase in fatalities: an analysis of United Kingdom national anaphylaxis data, 1992-2012. J Allergy Clin Immunol. (2015) 135:956-63.e1. doi: 10.1016/j.jaci.2014.10.021

9. Campbell RL, Hagan JB, Li JT, Vukov SC, Kanthala AR, Smith VD, et al. Anaphylaxis in emergency department patients 50 or 65 years or older. Ann Allergy Asthma Immunol. (2011) 106:401-6. doi: 10.1016/j.anai.2011.01.011

10. Lieberman P, Simons FE. Anaphylaxis and cardiovascular disease: therapeutic dilemmas. Clin Exp Allergy. (2015) 45:1288-95. doi: 10.1111/cea.12520

11. Simons FE, Ebisawa M, Sanchez-Borges M, Thong BY, Worm M, Tanno LK, et al. 2015 update of the evidence base: World Allergy Organization anaphylaxis guidelines. World Allergy Organ J. (2015) 8:32. doi: 10.1186/s40413-015-0080-1

12. Muraro A, Roberts G, Worm M, Bilò MB, Brockow K, Fernández Rivas M, et al. Anaphylaxis: guidelines from the European Academy of Allergy and Clinical Immunology. Allergy. (2014) 69:1026-45. doi: 10.1111/all.12437

13. Worm M, Francuzik W, Renaudin JM, Bilo MB, Cardona V, Scherer Hofmeier $\mathrm{K}$, et al. Factors increasing the risk for a severe reaction in anaphylaxis: an analysis of data from The European Anaphylaxis Registry. Allergy. (2018) 73:1322-30. doi: 10.1111/all.13380

14. Lee S, Hess EP, Nestler DM, Bellamkonda Athmaram VR, Bellolio MF, Decker WW, et al. Antihypertensive medication use is associated with increased organ system involvement and hospitalization in emergency department patients with anaphylaxis. J Allergy Clin Immunol. (2013) 131:1103-8. doi: 10.1016/j.jaci.2013.01.011

15. Harduar-Morano L, Simon MR, Watkins S, Blackmore C. Algorithm for the diagnosis of anaphylaxis and its validation using population-based data on emergency department visits for anaphylaxis in Florida. J Allergy Clin Immunol. (2010) 126:98-104. doi: 10.1016/j.jaci.2010.04.017
16. Sampson HA, Mendelson L, Rosen JP. Fatal and near-fatal anaphylactic reactions to food in children and adolescents. N Engl J Med. (1992) 327:380-4. doi: 10.1056/NEJM199208063270603

17. Wang J, Young MC, Nowak-Wegrzyn A. International survey of knowledge of food-induced anaphylaxis. Pediatr Allergy Immunol. (2014) 25:644-50. doi: $10.1111 /$ pai.12284

18. Worm M, Edenharter G, Ruëff F, Scherer K, Pföhler C, Mahler V, et al. Symptom profile and risk factors of anaphylaxis in Central Europe. Allergy. (2012) 67:691-8. doi: 10.1111/j.1398-9995.2012.0 2795.x

19. Grabenhenrich LB, Dölle S, Ruëff F, Renaudin JM, Scherer K, Pföhler $\mathrm{C}$, et al. Epinephrine in severe allergic reactions: the European Anaphylaxis Register. J Allergy Clin Immunol Pract. (2018) 6:1898-906.e1. doi: 10.1016/j.jaip.2018.02.026

20. Ring J, Beyer K, Biedermann T, Bircher A, Duda D, Fischer J, et al. Guideline for acute therapy und management of anaphylaxis. S2 guideline of DGAKI, AeDA, GPA, DAAU, BVKJ, ÖGAI, SGAI, DGAI, DGP, DGPM, AGATE and DAAB. Allergo J Int. (2014) 23:96-112. doi: 10.1007/s40629-01 4-0009-1

21. Truhlár A, Deakin CD, Soar J, Khalifa GEA, Alfonzo A, Bierens JJLM, et al . European resuscitation council guidelines for resuscitation 2015Section 4. Cardiac arrest in special circumstances. Resuscitation. (2015) 95:148-201. doi: 10.1016/j.resuscitation.2015.07.017

22. Clark S, Wei W, Rudders SA, Camargo CA Jr. Risk factors for severe anaphylaxis in patients receiving anaphylaxis treatment in US emergency departments and hospitals. J Allergy Clin Immunol. (2014) 134:1125-30. doi: 10.1016/j.jaci.2014.05.018

Conflict of Interest Statement: The authors declare that the research was conducted in the absence of any commercial or financial relationships that could be construed as a potential conflict of interest.

Copyright (C) 2019 Aurich, Dölle-Bierke, Francuzik, Bilo, Christoff, Fernandez-Rivas, Hawranek, Pföhler, Poziomkowska-Gęsicka, Renaudin, Oppel, Scherer, Treudler and Worm. This is an open-access article distributed under the terms of the Creative Commons Attribution License (CC BY). The use, distribution or reproduction in other forums is permitted, provided the original author(s) and the copyright owner(s) are credited and that the original publication in this journal is cited, in accordance with accepted academic practice. No use, distribution or reproduction is permitted which does not comply with these terms. 